\title{
Disaster Risk Research Literature on Statistics Analysis in China Journal Net
}

\author{
Shuzhen Li, Alateng Tuya* \\ College of Geographical Science, Inner Mongolia Normal University, Hohhot 010022, China, \\ E-mail:920194467@qq.com,altty@imnu.edu.cn
}

Received 11 April 2015

Accepted 24 May 2015

\begin{abstract}
Disaster risk literature collect from CNKI, analysis of the development of china journal net's literature on disaster risk. According to the literature was published in the year, subject, author, literature sources, databases, was cited and downloaded several aspects, such as for quantitative analysis. Statistical analysis of the literature on disaster risk can draw the following result: Risk of disaster in China started late until the beginning of 2007, the increasing number of documents; Domestic disaster risk leading university research station in Nanjing Information Engineering University, the leading journal is Disaster Reduction in China; Domestic cooperation on disaster risk degree of harmony with the rate of increase in value more, gradually in-depth research; Core journal literature citations high, indicating that field has a central effect, receive attention in academia.
\end{abstract}

Keywords: Disaster Risk; Bibliometrics method; CNKI

\section{中国期刊网灾害风险文献统计分析}

\section{李淑珍，阿拉腾图娅}

内蒙古师范大学地理科学学院, 内蒙古 呼和浩特 010022, 中国

\begin{abstract}
摘要: 从中国知网搜集灾害风险研究的文献, 分析国内期刊网灾害风险研究文献的发展。使用文献计量法, 对检索到的灾害风险文献, 按文献发表年份、题目、作者、文献来源、数据库、文献被引及下载频数等方 面来进行量化分析。统计分析灾害风险研究文献可以得出以下结果: 灾害风险研究在国内起步晚, 直到 2007 年开始文献数量迅速增多; 国内对灾害风险研究站领先地位的大学是南京信息工程大学, 期刊是中国减灾; 国内对灾害风险研究作者合作度和合著率数值越来越增高, 可知该研究领域发表文献质量越来越提高、研 究内容逐渐深入; 核心期刊文献被引频次高, 表示该领域中具有核心效应, 在学术界得到重视。
\end{abstract}

关键词: 灾害风险; 文献计量法; 中国知识资源总库

项目基金：国家自然基金项目（41261099）资助

作者简介: 李淑珍 (1991一), 女, 内蒙古人, 硕士研究生, 研究方向: 自然地理学、自然灾害研究

*通信作者: 阿拉腾图娅 (1969-), 女, 教授, 从事资源环境遥感研究 


\section{前言:}

由于现代社会人口不断增加、物质财富不断增多, 灾害的后果日益严重, 使我国涌出大量的研究员对灾 害风险进行研究, 灾害风险研究文献数量不断增多。 科学文献的数量是衡量科学知识的重要尺度之一, 科 学知识量的增长及其规律与科学文献的增长及其规律 是紧密联系的, 灾害风险文献的数量变化可以反映该 科学领域知识量的变化情况。文献计量方法可以分析 某一个科学的产生、发展、分化和相互渗透的动向; 可以确定重点文献、核心作者及核心期刊, 为文献报 道和指导读者重点阅读提供最佳方案; 可以了解研究 科学家的分布, 评价作者名望和识别科学人才。

文献计量学的发展大致分为三个阶段, 萌芽阶段 (1917-1933 年)、尊定时期（1934-1960 年）、全面发 展与分化时期 (1960 年至今) ${ }^{[1]}$ 。20 世纪初叶, 人们 已经开始对文献进行定量化研究, 但当时文献计量法 并没有作为一门独立的学科而存在, 它源于社会科学 借用自然科学研究的方法 ${ }^{[2]}$, 经过数十年的发展形成 一个独特的研究方法体系。1969 年英国著名情报学家 阿伦- 普里查德 (Alan Pritchard) 首次提出用术语 “Bibliometrics”取代“统计目录学”, 这一术语的出现标 志着文献计量学的正式诞生 ${ }^{[3]}$ 。直到 80 年代, 随着量 化方法在科研评价中的应用, 科研评价也经历了从专 家定性评价到定量评价相结合的过程, 文献计量方法 在科研评价中的作用逐渐受到重视, 它是图书馆学情 报学等领域的方法论体系中的重要组成。

文献计量法是主要由文献计量特征、文献统计分 析的方法、基本流程与文献计量规律几个部分构成 ${ }^{[4}$ ${ }^{5]}$, 以信息的外部特征为研究对象、以定量分析为出发 点, 在科学研究的诸多领域中解决了许多实际问题, 得到了社会的普遍认可。近年来, 我国发生的灾害的 数量与造成的损失急剧增加, 灾害已经是阻碍社会和 经济稳定发展的一个主要因素, 同时研究人员对灾害 风险的研究也逐渐深入, 为了使灾害风险研究人员迅 速掌握该领域的研究动态, 笔者用文献计量法对国内
期刊网灾害风险文献进行统计分析, 为进一步研究灾 害风险提供基本数据。通过文献统计分析, 了解国内 期刊网灾害风险研究的演进历程, 影响力较高的研究 员, 已经扩展的范围及发展趁势。

\section{1. 研究方法}

\section{1. 获取文献资源}

文献检索时间是 2014 年 11 月, 以中国知识资源 总库 (中国知网 CNKI) 为文献信息获取平台, 检索类 型为以“主题”为检索项, 以“灾害风险”为检索词，共 检索到 3912 篇文献。其中, 期刊文献有 2739 篇, 硕 士论文有 568 篇, 博士论文有 179 篇, 会议论文有 416 篇, 学术辑刊文献有 10 篇。且索取每篇文献的题名、 作者、来源、发表时间、数据库、被引及下载次数等 相关信息。再将搜索到的文献手工复制到 Excel 表格 中, 并进行整理, 这种方式虽然繁琐, 但收集的文献 比较全面。

\section{2. 数据库的选取原因}

中国期刊网即有优势也有弊端, 本文章主要依靠 它的优势来进行撰写的。首先，与国际三大检索系统 《科学引文索引》一 SCI、《工程索引》一 EI、《科技会 议录索引》-ISTP 比较可以知道, 虽然很多国家把三 大检索系统作为评价高等院校、科研机构的科研和学 术水平的标准, 但它们不收录中文期刊。中国期刊网 的大量汉语文献, 为中国众多的学者提供知识互动平 台, 为国内学术领域的发展提供了基础。其次, 与国 内其它数据库检索功能相比的话, 中国期刊网也有着 自己不可代替的一面。比如, 中国期刊网提供的检索 字段比维普和万方多, 它除了提供一些必须的检索字 段以外还提供了参考文献、全文、智能检索、中途分 类号、ISSBN 等字段, 便于更准确的检索到所需要的 文献, 并且中国期刊网还能进行引文查询等优势。 


\section{3. 文献数量分析与统计法}

对检索到的 3912 篇文献, 按学术期刊、硕士论 文、博士论文、会议论文和学术辑刊统计分类。根据 《2014 年北大核心期刊目录》将学术期刊分成普通期 刊与核心期刊。将检索到灾害风险文献采用文献计量 分析法, 利用 Excel 2010、origin 8.0 等进行统计分析。 按研究需求统计文献数量的年际分布、各期刊的载文 量、各大学发表的硕博士论文数量、核心作者群、被 引及被下载次数等。统计核心作者群时, 根据文献计 量学的洛特卡定律 ${ }^{[6]}$ 以第一作者为统计对象, 根据普 赖斯（D.Price）提出的计算公式确定核心作者群数量 [7]:

$$
\mathrm{M}=0.749\left(\mathrm{~N}_{\max }\right)^{1 / 2}
$$

公式中 $M$ 为论文篇数, $N_{\text {max }}$ 为所有统计文献里发表文 献数量最多的作者的总发表文献数量, 即发表文献数 量 M 以上的作者称为核心作者群。

统计文献被引用速度时利用加菲尔德 (E Garfield) 提出的即年指标测度。计算公式:

$$
\text { 即年指标 }=\frac{\text { 当年发表的文献被引用当年文献的篇数 }}{\text { 当年文献总数 }}
$$

\section{2. 统计分析结果}

\section{1. 灾害风险研究文献数量的阶段分析}

文献量是指某一学科研究者在某一段时间内所发 表的论文数量的多少。文献量的多少从一定程度上反 映着该学科的发展水平, 因此统计分析中国期刊网灾 害风险研究文献的数量来衡量该研究领域的发展动 态。统计 1983-2014 年的中国期刊网灾害风险文献数 量变化, 了解我国灾害风险研究领域的发展演进。从 图 1 可以看出, 我国期刊网灾害风险的研究可大致分 为初期、发展、兴旺阶段。

1983-2000 年为初期阶段, 文献量极少, 年均文 献数量为 7.2 篇。其中 1983-1986 年均只发表 1 篇文 献，且未检索到 1988、1989 年的相关文献。最早的一
篇文献是多萝西·内尔金发表在《科学对社会的影响》 上的《谈风险的社会和政治认可》, 它是在中国期刊网 上首次发表的灾害风险研究文献。在初期阶段研究文 献主要以风险、灾害及自然灾害等的理论概述为主, 但未形成一个众多研究者认同的统一理论概念。被后 者参考引用的次数最多的是黄崇福发表在核心期刊 《自然灾害学报》上的《自然灾害风险分析的基本原 理》和《以历史灾情资料为依据的农业自然灾害风险 评估方法》。

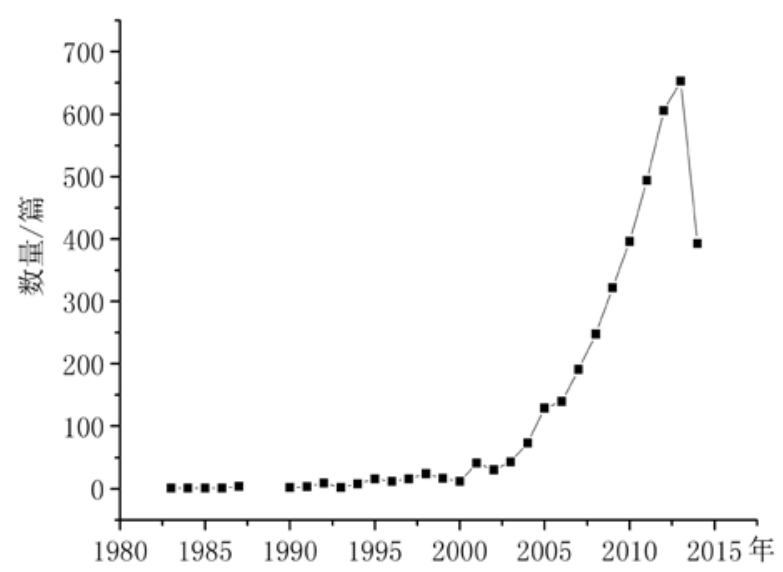

图 1 灾害风险文献数量年际变化

Fig.1. Annual variations of the published amounts of articles involved the disaster risk

2000 年开始全球范围内广泛开展“国际减灾战略” (International Strategy for Disaster Reduction, ISDR), 整体上改变了前者的减灾观点, 从灾后的反应转变为 灾前防御, 推动了学者们对灾害风险的研究。 2001-2006 年的发展阶段。此阶段文献量缓慢波动上 升, 到 2006 年达到 140 篇, 发展阶段年均文献量为 76 篇, 文献研究方向逐渐多样化, 内容从单一的理论 研究推广到多种方法研究、及广泛到不同区域尺度上 研究。

由于 2007 年发布《国家综合减灾“十一五”规划》 和 2008 年百年奥运会的到来对中国灾害风险研究有 
着很大的推动作用, 2007-2013 年为兴旺阶段。从 2007 年开始中国期刊网灾害风险文献年均数量急速增 长, 发展速度迅速上升, 2013 年文献数量达到最多 653 篇, 兴旺阶段年均文献数量为 415.7 篇。2014 年 文献数量为 393 篇, 因为未能检索到全年的文献数量, 所以在文献数量年即变化图上有下滑现象。国内经过 数十年的灾害风险研究中, 研究者们对风险、灾害风 险及自然灾害风险等名词有了较统一的认识。比如, 自然灾害风险的统一概念逐渐浮现, 它是由自然事件 或力量为主因导致的未来不利事件情景 ${ }^{[8]}$ 。

初步阶段文献数量少, 研究方向也不多, 经过发 展阶段和兴旺阶段之后, 文献数量不断增加, 研究方 向也逐渐增多。总的来说，1983-2013 年间中国期刊
网灾害风险研究文献数量的不断增加表示我国对灾害 风险领域的研究呈现越来越强劲的势头。

\section{2. 按文献类型统计分析}

\subsection{1 硕博士学位论文统计分析}

灾害风险研究领域的发展给国内众多硕博士带 来了很多科研机遇, 硕博士学位论文发表数量可以反 映灾害风险分析领域的发展现状。统计 1983-2014 年 在中国期刊网上发表的灾害风险硕博士学位论文, 最 早是 1992 年北京师范大学的黄崇福的博士论文, 题名 为《信心扩散原理与计算机思维及其在地震工程中的 利用》。硕士论文最早是 1999 年大连理工大学的郝军, 文献名为《石化企业投资、灾害风险与安全效益研究》。

表 1 . 发表论文数量 10 篇或以上的大学

Table 1 . Ten or above ten papers were publishes in the universities

\begin{tabular}{|c|c|c|c|c|}
\hline 排序 & 学位授予单位 & 硕士论文 (篇) & 博士论文（篇） & 总数（篇） \\
\hline 1 & 南京信息工程大学 & 44 & 6 & 50 \\
\hline 2 & 华东师范大学 & 18 & 11 & 29 \\
\hline 3 & 兰州大学 & 25 & 3 & 28 \\
\hline 4 & 成都理工大学 & 18 & 9 & 27 \\
\hline 5 & 长安大学 & 20 & 7 & 27 \\
\hline 6 & 北京师范大学 & 12 & 10 & 22 \\
\hline 7 & 中国地质大学 (北京) & 16 & 5 & 21 \\
\hline 8 & 西南财经大学 & 19 & 1 & 20 \\
\hline 9 & 中国海洋大学 & 11 & 8 & 19 \\
\hline 10 & 中南大学 & 13 & 3 & 16 \\
\hline 11 & 西南大学 & 12 & 3 & 15 \\
\hline 12 & 重庆大学 & 10 & 4 & 14 \\
\hline 13 & 大连理工大学 & 9 & 4 & 13 \\
\hline 14 & 东北师范大学 & 12 & 1 & 13 \\
\hline 15 & 上海师范大学 & 10 & 2 & 12 \\
\hline 16 & 华中农业大学 & 6 & 5 & 11 \\
\hline 17 & 天津大学 & 10 & 1 & 11 \\
\hline 18 & 中国农业科学院 & 5 & 5 & 10 \\
\hline 19 & 浙江大学 & 6 & 4 & 10 \\
\hline 20 & 首都师范大学 & 9 & 1 & 10 \\
\hline
\end{tabular}


之后各大学陆续发表, 直到 2014 年灾害风险硕士博士 论文文献总数量达到 747 篇。

在 3912 篇灾害风险文献中硕博士论文有 747 篇, 约占总文献的 $19 \%$ 。其中硕士论有 568 篇、博士论文 有 179 篇, 比例为约 $3: 1$, 分布于 169 所学校。根据 《学位授予单位》统计得出发表硕博士论文在 10 篇或 以上的学校有 20 所, 文献发表数量共 378 篇, 占总硕 博士论文的 $51 \%$ 。列出这些大学的名称和发表文献数 量见表 1 。

南京信息工程大学以 50 篇的总发量在中国期刊
网硕博士论文中处在领先地位, 发文献量约占总量 $6.7 \%$, 在所有大学中发表硕士论文量为最多共 44 篇, 博士论文为 6 篇。另外发文较多的有华东师范大学共 29 篇, 其中硕士论文有 18 篇, 博士论文有 11 篇, 所 有大学中发表博士论的文数量为最多。其次, 兰州大 学有 28 篇、成都理工大学和长安大学有 27 篇、北京 师范大学有 22 篇、中国地质大学（北京）有 21 篇、 西南财经大学有 20 篇。还有中国海洋大学、中南大学、 西南大学、重庆大学、东北师范大学、大连理工大学、 上海理工大学、天津大学、华中农业大学、首都师范

\section{表 2. 期刊刊名及载文量}

Table 2. Name of periodicals and the amount of downloading

\begin{tabular}{|c|c|c|c|c|}
\hline 排序 & 普通期刊名 & 载文量（篇） & 核心期刊名 & 载文量 (篇) \\
\hline 1 & 中国减灾 & 297 & 自然灾害学报 & 142 \\
\hline 2 & 中国应急管理 & 33 & 灾害学 & 94 \\
\hline 3 & 中国农学通报 & 29 & 中国安全科学学报 & 23 \\
\hline 4 & 安徽农业科学 & 28 & 地理科学进展 & 21 \\
\hline 5 & 中国地质灾害与防治学报 & 28 & 地质通报 & 19 \\
\hline 6 & 城市与减灾 & 24 & 中国农业气象 & 17 \\
\hline 7 & 防灾科技学院学报 & 23 & 北京师范大学学报 & 15 \\
\hline 8 & 贵州气象 & 20 & 资源科学 & 14 \\
\hline 9 & 中国防汛干旱 & 17 & 地理科学 & 14 \\
\hline 10 & 防灾博览 & 16 & 地理研究 & 13 \\
\hline 11 & 工程地质学报 & 16 & 气象 & 13 \\
\hline 12 & 地质灾害与环境保护 & 14 & 中国人口.资源环境 & 13 \\
\hline 13 & 气象科技 & 13 & 气候与环境研究 & 12 \\
\hline 14 & 气象研究与应用 & 13 & 山地学报 & 12 \\
\hline 15 & 中国民政 & 13 & 地球科学进展 & 11 \\
\hline 16 & 中国水利 & 12 & 水文地质工程地质 & 11 \\
\hline 17 & 海洋开发与管理 & 11 & & \\
\hline 18 & 气象与减灾研究 & 11 & & \\
\hline 19 & 现代农业科技 & 11 & & \\
\hline 20 & 气象与环境科学 & 10 & & \\
\hline
\end{tabular}


大学、浙江大学及中国农业科学院等大学的文献量均 少于 20 篇。

其余 149 所学校的发表文献量有 369 篇, 占总硕 博士总论文 $49 \%$ 。其中 67 所大学只有发表 1 篇论文、 30 所大学发表 2 篇论文、18 所大学发表 3 篇论文、 11 所大学发表 4 篇、 7 所大学 5 篇论文、 8 所大学 6 篇论 文、 5 所大学 7 篇论文、3 所大学发表 9 篇论文。

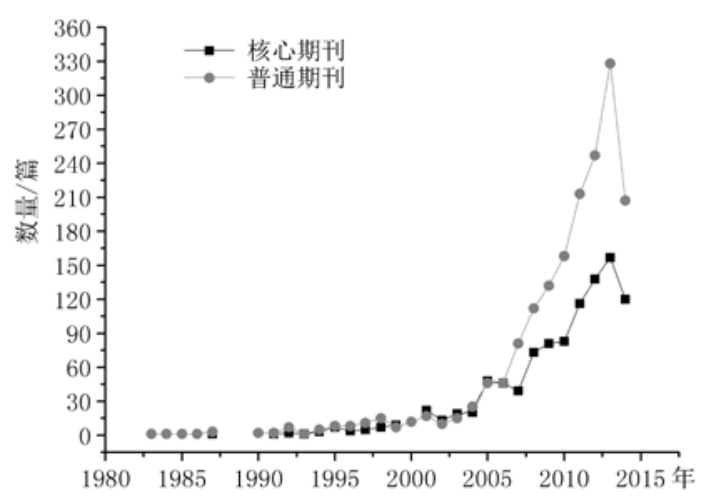

图 2. 灾害风险期刊文献数量的年际分布

Fig.2. Number of annual distribution of disaster risk journal literature

\section{2 .2 统计分析期刊文献}

在学术界里核心期刊和普通期刊的作用和意义有 很大的区别, 核心期刊是学术期刊质量评判和论文学 术水平鉴定的主要依据, 核心期刊文献是热点研究问 题, 因此按《2014 年北大核心期刊目录》统计分类 2739 篇灾害风险期刊文献, 其中核心期刊文献有 1017 篇, 普通期刊文献有 1722 篇。图 2 是 1983-2014 年间的 中国期刊网期刊文献分布。

1993、2005 年普通期刊与核心期刊的文献发表数
量相等，在 1999、2001、2003、2005 年核心期刊上的 文献发表数量较多, 但相差不大, 见图 2。从 2007 年 开始普通期刊文献发表数量急速增长, 年均文献发表 量远超核心期刊文献发表量。普通期刊从 2008 年开始 年文献发表数量达到 100 篇以上, 核心期刊从 2011 年 开始年文献发表量达到 100 篇以上。2013 年是期刊文 献达到顶峰时期, 普通期刊文献在达到 328 篇, 核心 期刊文献达到 157 篇。最早发表是 1983 年在《科学对 社会的影响》上的普通期刊文献, 核心期刊文献最早 发表是 1987 年在《金融与经济》上。总的来说, 2007 年开始期刊文献增加速度比较明显, 核心期刊文献数 量也随之增加。可见随着国家建设对灾害风险研究的 需求不断增加, 在众多地理学界学者、专家的支持和 培育下, 高质量的论文越来越多, 灾害风险得到逐步 发展和进步。

核心期刊文献年发表数量增加, 但远远比不过普 通期刊的数量增加速度。有两个原因, 一个原因是在 核心期刊上发表的论文档次需求比较高, 所以文献发 表难度较高。还有一个原因是核心期刊种类比普通期 刊种类少。统计 1983-2014 年间的中国期刊网总期刊 种类数量及每个期刊的载文量可知, 2739 篇期刊文献 共有 953 种期刊上发表, 其中普通期刊有 672 个, 载 文量有 1722 篇。核心期刊有 281 个, 载文量有 1017 篇。

比较载文量在 10 或以上的核心期刊与普通期刊, 见表 2 。核心期刊有 16 个, 载文量为 444 篇, 占核心 期刊文献的 44\%。普通期刊有 20 个, 载文量有 639 篇, 占普通期刊文献的 37\%。中国减灾以 297 篇的载 文量在中国期刊网灾害风险研究领域中占领先位置,

表 3. 对比核心期刊与普通期刊被下载次数

Table 3. Compare of core periodicals and common periodicals in the amount of downloading

\begin{tabular}{lccccccc}
\hline 被下载次数 & $3000 \sim 2500$ & $2500 \sim 2000$ & $2000 \sim 1500$ & $1500 \sim 1000$ & $1000 \sim 500$ & $500 \sim 0$ & 0 \\
\hline 核心期刊 & 2 & 3 & 13 & 32 & 137 & 815 & 15 \\
普通期刊 & 0 & 0 & 1 & 1 & 43 & 1606 & 71 \\
\hline
\end{tabular}


其次有, 自然灾害学报、灾害学报。普通期刊里, 中 国减灾载文量最多, 有 297 篇, 占普通期刊文献的 $17.2 \%$, 其次是中国应急管理、中国农学通报等。核 心期刊里, 自然灾害学报的载文量最多, 有 142 篇, 占核心期刊文献的 14\%、其次是灾害学、中国安全科 学学报等。
核心期刊作为重要期刊, 得到很多研究员的关注, 统计 1983-2014 年间的中国期刊网期刊文献的被下 载次数, 见表 3 。统计比较中国期刊网灾害风险核心 期刊文献与普通期刊文献的被下载次数, 被下载次数 越的文献表示在灾害风险研究领域中越受关注, 影响 力越大。从表 3 可以看出, 被下载 2000 次以上的核心

表 4. 灾害风险总文献核心作者及文献篇数

Table 4. Amount of core authors and literatures in the whole literature about disaster risk

\begin{tabular}{ccc}
\hline 人数 & 文献第一作者 & 50 \\
\hline 1 & 篇数 \\
\hline 1 & 金磊 & 33 \\
1 & 史培军 & 19 \\
1 & 徐娜 & 17 \\
2 & 黄崇福 & 13 \\
2 & 肖志强、扈海波 & 11 \\
2 & 张继权、刘希林 & 9 \\
5 & 尚志海、刘传正 & 7 \\
10 & 张俊香、张春山、萍、赵思建、殷杰、王绍玉、刘兰芳 & 6 \\
24 & 朱良峰、朱传林、郑慧、赵州、张文宗、张庆阳、叶欣梁、王志涛、王禹、王东明、 & 5 \\
& 唐亚明、任鲁川、彭贵芬、潘东华、娄伟平、刘敏、刘丽、刘家福、李瑞昌、李红 & \\
& 英、黄慧、胡宝清、杜军、丁文广 & \\
\hline
\end{tabular}

表 5. 灾害风险核心期刊的核心作者群及篇数

Table 5. Amount of core authors and articles in the core periodicals about disaster risk

\begin{tabular}{ccc}
\hline 人数 & 文献第一作者 & 篇数 \\
\hline 1 & 史培军 & 20 \\
1 & 黄崇福 & 14 \\
1 & 刘希林 & 9 \\
1 & 金磊 & 8 \\
3 & 张继权、刘传正、扈海波 & 6 \\
1 & 李红英 & 5 \\
10 & 赵思建、殷杰、薛晔、吴益平、王志涛、苏筠、尚志海、罗培、刘丽、崔鹏 & 3 \\
25 & 周洪建、张俊香、张竟竟、张春山、殷坤龙、谢全敏、唐亚明、唐川、孙才志、石菊 & 3 \\
& 松、任鲁川、潘东华、倪长健、刘新立、李谢辉、金菊良、金江军、蒋卫国、胡培培、 & \\
& & 韩用顺、葛怡、杜鹏、丁文广、陈香、陈鹏 \\
\hline
\end{tabular}


期刊有 5 篇, 普通期刊未被下载过。被下载 2000 1500、 1500 1000、普通期刊未被下载过。被下载 2000 1500、 1500 1000、1000 500 次的核心期刊依次有 13、32、 137 篇, 普通期刊依次有 $1 、 1 、 43$ 篇。表示灾害风险 论文的核心期刊文献的被下载次数比较多, 学术界、 社会的关注度高。被下载 500 次以下的核心期刊文献 与普通期刊文献均集中在近几年, 表示文献被下载具 有时间滞后特点。

\section{3. 对文献作者进行统计分析}

\subsection{1. 统计文献的核心作者群}

作者是期刊论文的重要外部特征之一，是决定期 刊论文质量的关键; 而核心作者更是期刊质量稳固和 继续发展的坚实基础 ${ }^{[9]}$ 。统计比较中国期刊网灾害风 险总文献核心作者群和核心期刊文献核心作者群, 从 灾害风险总文献中统计出共有 2584 位第一作者, 核心 期刊文献中统计出共有 757 位第一作者。根据文献计 量学的洛特卡定律以第一作者为统计对象, 把 $\mathrm{N}_{\text {max }}$ 总 $=50$ 和 $N_{\max }$ 核 $=20$ 代入式 (1) 中, 以取整的原则得出
$M_{\text {总 }}=5$ 和 $M$ 总 $=3$, 即发表文献在 5、3 篇以上的作者为 中国期刊网灾害风险总文献与核心期刊文献的核心作 者群，见表 4 和表 5 。

比较表 4 和表 5 看出, 灾害风险总文献核心作者 共有 49 位, 核心期刊核心作者共有 43 位, 其中史培 军、黄崇福、金磊、扈海波、张继权、刘希林等 24 位 作者相互重叠, 并且这些作者的文献发表数量均在领 先位置, 表示核心期刊核心作者群也是总文献的核心 作者群，核心期刊及核心期刊作者在该研究领域中具 有核心效应。

灾害风险研究总文献核心作者群的文献总发数量 400 篇, 占总文献 $10 \%$ 。核心期刊核心作者群的文献 总发数量 189 篇, 占核心期刊总文献 19\%。这与普赖 斯定律 50\%相差太远，表示核心作者发表的文献量极 少, 在文献计量角度上核心作者群还未形成, 需要更 多的研究人员来领头研究灾害风险研究。

\subsection{2. 作者合作度与合著率}

表 6. 作者发文量与合作度

Table 6. Amount of publishment by the author and cooperation

\begin{tabular}{|c|c|c|c|c|c|c|c|c|c|}
\hline \multirow[t]{2}{*}{ 年份 } & \multicolumn{5}{|c|}{ 发文作者与论文篇数（人/篇） } & \multirow{2}{*}{\multicolumn{2}{|c|}{ 人次 }} & \multirow[t]{2}{*}{ 合作度 } & \multirow[t]{2}{*}{ 合著率 \% } \\
\hline & 1 & 2 & 3 & 4 & $\geq 5$ & & & & \\
\hline 1983-1986 & 2 & & 2 & & & 4 & 8 & 4 & 50 \\
\hline 1987-1990 & 3 & 3 & & & & 6 & 12 & 1.5 & 50 \\
\hline 1991-1994 & 11 & 9 & 2 & & & 22 & 35 & 1.6 & 50 \\
\hline 1995-1998 & 34 & 13 & 8 & 2 & 1 & 58 & 97 & 1.7 & 41 \\
\hline 1999-2002 & 46 & 21 & 12 & 8 & 8 & 95 & 197 & 2.1 & 51.6 \\
\hline $2003-2006$ & 157 & 152 & 69 & 33 & 44 & 455 & 1026 & 2.3 & 65.5 \\
\hline $2007-2010$ & 515 & 157 & 154 & 125 & 104 & 1055 & 2378 & 2.6 & 51.2 \\
\hline $2010-2014$ & 826 & 319 & 307 & 220 & 272 & 1944 & 4784 & 2.5 & 57.5 \\
\hline 平均 & & & & & & & & 2.3 & 52.1 \\
\hline 合计 & 1594 & 674 & 554 & 388 & 429 & 3639 & 8537 & & \\
\hline 比例 & 43.8 & 18.5 & 15.2 & 10.7 & 11.8 & 100 & & & \\
\hline
\end{tabular}


随着对合作者问题研究的逐渐深入, 规定了合作 度和合著率两个计量指标来反映某科学领域内作者合 作智能的发挥程度, 数值越高, 合作智能发挥越充分, 表示研究越深入、文献质量越高。美国学者 D.deB Beaver 等在《科学合作研究》这篇论文上通过科学论 文合著的文献计量学方法论证了科学合作研究的客观

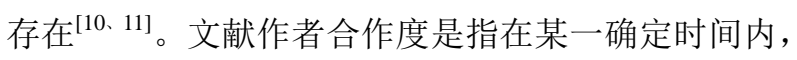
每篇文献的平均作者数 ${ }^{[12]}$ 。文献合著率是指在某一确 定时间内, 合著文献在总文献中的百分比量。

分析中国期刊网灾害风险文献在 1984-2014 年 间作者论文量、合作度和合著率的变化可以总结出以 下几个结果: 见表 6 可知, 单个作者的发文量为 1594 篇, 占 $43.8 \%$, 两个人及以上作者的发文量有 2045 篇, 占 $56.2 \%$, 合著的文献数量比单个作者文献数量较多。 说明随着现代科学的发展, 灾害风险研究的各种现代
险文献的作者合作成都还未达到很高的水平, 需要继 续增加作者合作成度来进一步完善文献质量。

\section{4 文献利用速度分析}

文献被引用的频次是衡量该科学领域交流程度和 作者吸收同领域研究进展的信息程度，它是科技文献 的重要组成部分, 也是衡量文献质量的一个重要指标。 从布拉德福定律 (Bradford law) 可知, 某一科学中的 多数论文及研究成果集中于该学科中少数的核心期刊 中 $^{[13]}$ 。在中国期刊网上, 统计 1983-2014 年间的核心 期刊、普通期刊, 博士论文、硕士论文及会议论文等 文献的被引用频次。表 7 中表示, 被引用文献在总文 献中的百分比、平均每篇文献的被引次数及最多被引 次数, 表示各类文献被引差距及各类文献的平均被引 次数。

\section{表 7. 文献被引比例}

Table 7. Percent of quotation by other literatures

\begin{tabular}{cccc}
\hline 文献类型 & 被引篇数 / 文献数量 & 被引总次数 / 文献数量 & 最多被引次数 \\
\hline 核心期刊 & $72 \%$ & 14.1 & 508 \\
博士论文 & $70 \%$ & 7.6 & 74 \\
硕士论文 & $44 \%$ & 1.8 & 20 \\
普通期刊 & $38 \%$ & 2.9 & 194 \\
会议论文 & $10 \%$ & 0.2 & 8 \\
\hline
\end{tabular}

化手段的应用、及学科间的交叉渗透, 文献作者开始 向群体联合研究方向发展; 平均合作度是 2.3 , 在 1987-1990 年为最少, 有 1.5, 之后期间数值一直提 高, 直到 2010-2014 年, 达到 2.3。平均合著率是 $52.1 \%$, 除了在 1995-1998 年间为最少, 有 $41 \%$, 其 他期间均在 50\%以上, 特别是在 2003-2006 年间达 到最高, 有 $65.5 \%$ 。表示中国期刊网灾害风险文献的 研究水平逐渐提高, 难度逐渐增大、深度逐渐增加; 从合作度和合著率的数量上来看, 中国期刊网灾害风
从表 7 可知，核心期刊的被引用最多，1017 篇核 心期刊的 $72 \%$ 曾经被引过，平均每篇核心期刊被引用 14.1 次。其次有，169篇博士论文的 70\% 曾经被引过， 平均每篇博士论文被引用 7.6 次。再次为硕士论文, 普通期刊, 会议论文等 (学术辑刊数量仅有 10 篇, 所 以被忽略)。被引次数在 200 次以上有 6 篇文献均为核 心期刊。有史培军的 《三论灾害研究的理论与实践》 被引 508 次; 第一作者为周成虎的《基于 GIS 的洪水 灾害风险区划研究》被引 315 次; 史培军的《四论灾 
害系统研究的理论与实践》被引 272 次; 黄崇福的《自 然灾害风险分析的基本原理》被引 250 次; 第一作者 为殷坤龙的《滑坡灾害空间区划及 GIS 应用研究》被 引 246 次; 第一作者是黄崇福的《以历史灾情资料为 依据的农业自然灾害风险评估方法》被引 215 次。其 中, 被引指标在微观评价中出现负面现象, 微观层次 评价是指对研究人员个人和期刊的评价 ${ }^{[14]}$ 。也有学者 为了提高自己论文的档次而盲目的引用名人的文献。

文献被引用的速度或时差是评价一个科学领域跟 踪最新研究动态的一个重要指标, 利用加菲尔德 $(\mathrm{E}$

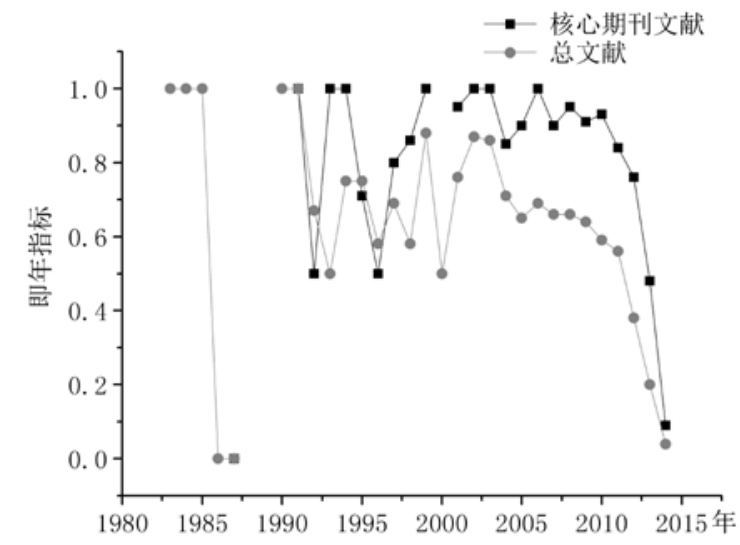

图 3. 1983-2014 年即年指标变化趁势

Fig. 3. A few years from 1983 to 2014 index trend

Garfield）提出的即年指标对灾害风险文献利用速度进 行测度。根据公式 2 计算 1983-2014 年灾害风险文献 的即年指标, 指标变化趁势如图 3 所示, 图中直线中 断表示某些年间未检索到相关文献。

在 1983 1991 年间, 由于文献数量过少, 灾害风 险总文献即年指标波动大, 接近于 1 或 0 , 走向两个 极端, 没有研究意义。1992-2011 年, 灾害风险总文 献从 10 篇左右增加到 500 篇左右, 随着总文献数量增 大被引用的文献数量也增加, 总文献平均即年指标为 0.68 ; 核心期刊平均即年指标是 0.87 , 其中未检索到 2000 年的核心期刊。2012-2014 年文献总发表数量增 加到顶端, 但从图 3 上看, 即年指标均不到 $0.5 ; 2013$ 、
2014 年核心期刊的即年指标也不到 0.5, 因为文献被 引用具有时间滞后特点。总的来说, 灾害风险文献中 核心期刊文献的被引用比较频繁, 核心期刊文献具有 核心效应, 核心期刊理论所使用的引用指标在一定程 度上也满足了研究人员对学术水平进行评价的需求。 相反, 低水平、低质量的科研论文, 是不大可能甚至 不会被引用的。

\section{3 结论}

基于 CNKI 文献数据库的文献统计分析, 分析 1983-2014 年的灾害风险研究的文献产出、研究的主 要领域和学科的主要特点等, 可以得出以下结论:

（1）中国期刊网灾害风险文献发展经过初步阶段 （1983-2000 年)、发展阶段（2001-2006 年）及兴 旺阶段（2007-2013 年）。从文献数量增加速度上看, 在 2007 年以前处于缓慢上升阶段, 之后开始快速上 升, 直到 2013 年文献发表数量达到顶峰值; 从研究内 容上来看, 起初发表文献方向单一, 随着灾害风险研 究领域的发展, 研究内容变得更加多样、全面、成熟。 总之它是一门新兴学科, 有现越来越强劲的发展势头。

(2) 统计硕博士论文得知, 南京信息工程大学论 文总发表数量占领先地位, 其中硕士论文数量在总硕 士论文中最多。华东师范大学的总发表数量文排第二, 其中博士论文是总博士论文中的最多。硕博士论文发 表比率为 3:1, 国内博士人数少, 对博士论文质量的要 求高, 发表难度较大。统计期刊文献得知, 中国减灾 的载文量在灾害风险研究领域中排第一，其次是核心 期刊自然灾害学报的载文量最多。我国核心期刊数量 比普通期刊少，但核心期刊文献质量高，具有核心作 用，被下载次数多。核心期刊近几年的平均被下载次 数较少，表示文献被下载具有时间滞后特点。

（3）统计中国期刊网灾害风险文献核心作者群可 知, 核心期刊文献的核心作者群在整个研究领域中具 有核心效应，关注度高。但用普赖斯定律计算可知， 核心作者发表的文献量少, 在文献计量角度上核心作 
者群还未形成, 需要更多的研究人员来领头研究灾害 风险研究。灾害风险研究领域的作者合作度和合著率 逐渐增加, 表示文献作者开始向群体联合研究方向发 展, 有利于产出高质量的文献。

（4）统计中国期刊网文献被引频次可知, 灾害风 险文献中核心期刊文献的被引用率最高, 核心期刊文 献质量高, 也是研究领域的热点问题。其次依次是博 士论文、硕士论文、普通期刊、会议论。根据际年指 标测度可知, 核心期刊即年指标数值比总文献几年指 标高, 均在 0.5 以上。但在 2012-2014 年文献即年指 标均不到 0.5 , 因为文献被引用具有时间滞后特点, 文 献被发表之后的短期间里不可能多次被引用。

\section{参考文献:}

[1] 赵蓉英, 徐丽敏.文献计量学发展演进与研究前沿 的知识图谱探析 [J]. 中国图书馆学 报,2010,36(189):061-064

[2] Zhao Rongying, Xu Limin. The Knowledge Map of the Evolution and Research Frontiers of the Bibliometrics[J]. Journal of Library Science in China, 2010, 36(189) :061-064

[3] 王曰芬, 文献计量法与内容分析法的综合研究[D]. 南京理工大学, 2007:1-47

Wang Yuefeng, A Synthetic Research of Bibliometric method and Content analysis method[D]. Nanjing University of Science and Technology, 2007: 1-47

[4] 邱均平.文献计量学[M]. 北京: 科学技术文献出版 社, 1988:1-40

Qiu Junping. Bibliometric[M]. Beijing: Science and Technology literature Press,1998:1-40

[5] 罗武胜, 文献计量学概论 [M].广州: 中山大学出版 社, 1994:2-36

Luo Wusheng. Introduction to Literature Metrology[M]. Guangzhou: Sun yat-sen University
Press, 1994: 2-36

[6] Élaine Gauthier. Bibliometric Analysis of Scientific and Technological Research: A User's Guide to Methodology[C]. Science and Technology Redesign Project Statistics Canada,1998.

[7] Lotka, A. j. The Frequency Distribution of Scientific Productivity, Journal of the Washington Academy of Science, 1962, 16(12):317-323

[8] 丁学东.文献计量学基础[M]. 北京: 北京大学出版 社,1993：204-232

Ding Xuedong. The Foundation of the Literature Metrology[M]. Beijing: Peking University Press,1993: 204-232

[9] 黄崇福.自然灾害风险分析与管理[M].北京：科学 出版社, 2012：43

Chongfu Huang. Analysis and Management of Natural Disaster Risk[M].Beijing: Science Press, 2012: 43

[10] 廉清, 《图书情报工作》核心作者群分析研究[J]. 现代情报，2004，（11）:55

Lian Qing,The analysis and Research of the Major Authors in 《Library and Information Service》 [J].Journal of Modern Information, 2004, (11) :55

[11] 金炬, 武夷山, 梁战平.国际科技合作文献计量学 研究综述—《科技计量学》(Scientometrics) 期 刊相关轮综述[J].图书馆情报工作，2007，52（3): 64

JinJu, Wu Yishan, Liang Zhangping. A Summary of Bibliometric Study on International Science and Technology Cooperation[J]. Library and Information Service, 2007, 52 (3): 64

[12] Beaver D deB, Rosen R. Studies in scientific collaboration: Part I : The professional origins of scientific co-authorship: Scientometrics, 1978, 1(1): 65-84 
[13] 安秀芬. 《图书情报工作工作》论文作者群的统 计分析 [J].图书馆情报，1994，(5）:29-30

An Xiufen. The Statistic Analysis of the Authors in 《Library and Information Service》[J]. Library and Information, 1994, (5): 29-30

[14] 杨廷郊, 马费城. 布拉德福定律的理论发展[J].情报 学刊, 1981, (4):61-62

Yang Tingjiao. Bradford Law's theory development. Journal of Information Science[J],1981，（4）:61-62

[15] 武夷山,梁立明.采用文献计量学指标进行科研绩 效量化评价应注意的几个问题[J]. 分析与评论, 2001,12(2): 110

Wu Yishan, Liang Liming. Some Problems about Should pay Attention to Use the Bibliometric Index to Proceed Scientific Research Performance Quantitative Evaluation[J]. Analysis and Comment, 2001,12(2):110 